PROCEEDINGS OF THE

AMERICAN MATHEMATICAL SOCIETY

Volume 130, Number 3, Pages 841-847

S 0002-9939(01)06067-1

Article electronically published on August 29, 2001

\title{
COMPLETING THE CONFORMAL BOUNDARY OF A SIMPLY CONNECTED LORENTZ SURFACE
}

\author{
ROBERT W. SMYTH
}

(Communicated by Wolfgang Ziller)

\begin{abstract}
This paper extends Kulkarni's conformal boundary $\partial \mathcal{L}$ for a simply connected Lorentz surface $\mathcal{L}$ to a compact conformal boundary $\partial^{c} \mathcal{L}$. The procedure used is analogous to Carathéodory's construction (in the definite metric setting) of prime ends from the accessible points of a bounded simply connected planar domain. The space $\partial^{c} \mathcal{L}$ of conformal boundary elements is homeomorphic to the circle, and contains Kulkarni's conformal boundary $\partial \mathcal{L}$ as a dense subspace.
\end{abstract}

\section{INTRODUCTION}

A Lorentz surface $\mathcal{L}=(S,[h])$ is an oriented, connected $C^{\infty}$ 2-manifold $S$ together with a conformal class of indefinite metrics on $S$. Such a conformal class of indefinite metrics determines a naturally ordered pair of smooth null direction fields $\mathcal{X}$ and $\mathcal{Y}$ on $S$, with $\mathcal{X}$ and $\mathcal{Y}$ distinct at each point of $S$. Local coordinates $x, y$ on $S$ are called proper null coordinates (with respect to $[h]$ ) if $\frac{\partial}{\partial x}$ lies along $\mathcal{X}$, $\frac{\partial}{\partial y}$ lies along $\mathcal{Y}$ and $h=2 B d x d y$ for some positive function $B$. The inextendible integral curves of $\mathcal{X}$ are referred to as X-lines and the inextendible integral curves of $\mathcal{Y}$ are called Y-lines. These two families of null lines capture the structure of $\mathcal{L}$. Although this structure is locally trivial, it has some interesting global properties. (See [12] or [5] for a much more thorough presentation.) For later reference, we note here that the span of a null line $\gamma$ is the set of all points on null lines which cross $\gamma$. The joint span of two null lines of opposite type (which meet each other) is the intersection of their spans.

There are stark contrasts between the consequences of conformality in the definite and indefinite metric cases. For example, the celebrated Koebe uniformization theorem shows that there are exactly 3 conformally distinct simply connected Riemann surfaces, but there exist uncountably many conformally distinct simply connected Lorentz surfaces. (See 9 or [10].) While $C^{1}$ conformal maps (and even 1-quasiconformal maps) between Riemann surfaces must be smooth, there exist $C^{j}$ conformal equivalences between Lorentz surfaces which are not $C^{j+1}$ conformally

Received by the editors April 17, 2000 and, in revised form, September 5, 2000.

2000 Mathematics Subject Classification. Primary 53C50, 53A30.

Key words and phrases. Indefinite metric, conformal geometry, foliation theory.

(C)2001 American Mathematical Society 
equivalent for $j=0,1, \ldots$ (See 8 .) Nevertheless, this paper develops a construction of conformal boundary elements for any simply connected Lorentz surface in a way which is closely analogous to Carathéodory's construction of prime ends. Several references are made in this paper to [7] in order to highlight the analogy. (All page number references to 7] refer to volume 3.) Alternatively, one may consult the treatment of Carathéodory's construction in [1] or [2].

\section{TOPOlOGical LOREnTZ SURFACES}

The natural correspondence between Lorentz surfaces and box surfaces can be readily adapted to provide a definition of a topological Lorentz surface. The detailed presentation from 12] of this correspondence is summarized below. Charts $\chi: U \rightarrow$ $\mathbb{R}^{2}$ and $\hat{\chi}: \hat{U} \rightarrow \mathbb{R}^{2}$ from the differentiable atlas $\mathcal{A}$ on $S$ are $C^{\square}$-related if $\hat{\chi} \circ \chi^{-1}$ may be expressed in some neighborhood of any point in $\chi(U \cap \hat{U})$ by functions $\hat{x}=f(x)$ and $\hat{y}=g(y)$ with $f^{\prime} g^{\prime}>0$. A box surface is an ordered pair $\left(S, \mathcal{A}^{\square}\right)$ where $\mathcal{A}^{\square}$ is a subatlas of $\mathcal{A}$ such that all charts of $\mathcal{A}^{\square}$ are $C^{\square}$-related and $\mathcal{A}^{\square}$ is maximal with respect to this condition. Given a box surface $\left(S, \mathcal{A}^{\square}\right)$ one can construct an indefinite metric $h$ on $S$ such that the charts in $\mathcal{A}^{\square}$ yield proper null coordinates on $S$ with respect to $[h]$. Conversely, given a Lorentz surface $\mathcal{L}=(S,[h])$ the collection of all charts that yield proper null coordinates forms a maximal $C^{\square}$ atlas on $S$. The most trivial example of a Lorentz surface is the Minkowski plane $E^{2,1}=\left(\mathbb{R}^{2},[d x d y]\right)$. The X-lines of the Minkowski plane are the horizontal lines and the Y-lines are the vertical lines. Among the box charts of the corresponding box surface are the maps obtained by restricting the identity on the plane to each open subset.

The definitions above may be adapted to the context of topological 2-manifolds merely by replacing the condition $f^{\prime} g^{\prime}>0$ by the condition $f$ and $g$ both strictly increasing or both strictly decreasing. The concept of a topological box surface which results may be used as the definition for a topological Lorentz surface. Let $\mathcal{L}=\left(S, \mathcal{A}^{\square}\right)$ be a topological Lorentz surface. The box surface characterizations in the differentiable setting of important Lorentz surface theory objects are here used as definitions. Coordinates on $S$ are proper null if they come from a chart in $\mathcal{A}^{\square}$. A locally one-to-one curve $\gamma: \mathcal{I} \rightarrow S$ is called null if for any $c \in \mathcal{I}$ and any $\chi \in \mathcal{A}^{\square}$ whose domain contains $\gamma(c)$, there exist $a, b \in \mathcal{I}$ such that $a<c<b$ and the image of $\left.\chi \circ \gamma\right|_{(a, b)}$ is a horizontal or vertical segment. An inextendible null curve which fits the horizontal (resp. vertical) case above at each point of its domain is called an Xline (resp. Y-line). (Here a curve which may be extended after reparameterization is considered extendible.) Two null lines which are reparameterizations of each other are sometimes treated as the same null line. A conformal homeomorphism from the topological Lorentz surface $\mathcal{L}_{1}=\left(S_{1}, \mathcal{A}_{1}^{\square}\right)$ to the topological Lorentz surface $\mathcal{L}_{2}=\left(S_{2}, \mathcal{A}_{2}^{\square}\right)$ is a homeomorphism from $S_{1}$ to $S_{2}$ which takes X-lines to X-lines and $\mathrm{Y}$-lines to Y-lines.

Many of the basic results about Lorentz surfaces can be shown to hold also for topological Lorentz surfaces with trivial modifications to statements and proofs. (E.g. Lemmas 3, 4 in [12.) Other basic results (e.g. Lemmas 11-15 in [12]) follow readily from the invariance of indices computed using Hamburger polygons constructed with null arcs. (See [3].) Of course any Lorentz surface $\mathcal{L}$ determines a topological Lorentz surface whose null lines are the same as those of $\mathcal{L}$ together with all $C^{0}$ reparameterizations thereof. 


\section{KULKARNI'S CONFORMAL BOUNDARY}

The following treatment of Kulkarni's conformal boundary differs in flavor somewhat from Kulkarni's original presentation in [5], but is nevertheless easily seen to amount to much the same thing.

Suppose $\mathcal{L}=\left(S, \mathcal{A}^{\square}\right)$ is a simply connected topological Lorentz surface. Given a null line $\gamma:(a, b) \rightarrow S$ and $c \in(a, b)$, the restrictions $\left.\gamma\right|_{(a, c]}$ and $\left.\gamma\right|_{[c, b)}$ are called end rays. If the traces $\operatorname{tr}(\alpha)$ and $\operatorname{tr}(\beta)$ of the end rays $\alpha$ and $\beta$ have noncompact intersection, then $\alpha$ and $\beta$ are said to have the same ideal endpoint. (Lemma 15 of [12] implies that two end rays that have the same ideal endpoint lie along the same null line.) End rays $\alpha$ and $\beta$ are called adjacent if there exists a chart $(U, \chi) \in \mathcal{A}^{\square}$ with $\operatorname{tr}(\alpha), \operatorname{tr}(\beta) \subset U$ such that $\chi(\operatorname{tr}(\alpha))$ and $\chi(\operatorname{tr}(\beta))$ are adjacent sides without the common vertex of some rectangle. Let $\sim$ be the equivalence relation on the collection of all end rays of $\mathcal{L}$ generated by the relations of adjacency and having the same ideal endpoint. The conformal boundary $\partial \mathcal{L}$ of $\mathcal{L}$ is the set of all equivalence classes of $\sim$. Clearly conformal maps respect $\sim$ so that a conformal map $f: \mathcal{L} \rightarrow \hat{\mathcal{L}}$ between simply connected Lorentz surfaces induces a well-defined map $\partial f: \partial \mathcal{L} \rightarrow \partial \hat{\mathcal{L}}$ given by $\partial f([\alpha])=[f(\alpha)]$ where $\alpha$ is any end ray in $\mathcal{L}$.

(The relation $\sim$ used here to form conformal boundary points as classes of end rays may be viewed as an indefinite metric analog of the relation used by Carathéodory to form accessible boundary points of a bounded simply connected planar domain as classes of Jordan half-intervals. See the definition on p. 52 and Theorem 2.9 of [7]. Note, however, that a conformal map between bounded simply connected subspaces of $E^{2}=\left(\mathbb{R}^{2},\left[d x^{2}+d y^{2}\right]\right)$ need not take accessible points to accessible points.) The rank of a conformal boundary point $P$ is equal to the largest possible number of representatives of $P$ no two of which have the same ideal endpoint.

Consider for example the topological Lorentz surface $\mathcal{L}_{\infty}=\left(\mathbb{R}^{2}, \mathcal{A}_{\infty}^{\square}\right)$ where the charts in $\mathcal{A}_{\infty}^{\square}$ include the complex exponential map restricted to each open subset of $\mathbb{R}^{2}$ on which it is one-to-one. For each $c \in \mathbb{R}$ and $k \in \mathbb{Z}$ the horizontal ray $\gamma_{c, k}:(-\infty, c] \rightarrow \mathbb{R}^{2}$ given by $\gamma_{c, k}(t)=\left(t, \frac{\pi k}{2}\right)$ is an end ray in $\mathcal{L}_{\infty}$. The end ray $\gamma_{c, k}$ lies along an $\mathrm{X}$-line if $k$ is even and along a Y-line if $k$ is odd. End rays $\gamma_{c, k}$ and $\gamma_{d, l}$ have the same ideal endpoint if and only if $k=l$. The end rays $\gamma_{c, k}$ and $\gamma_{d, k+1}$ are adjacent. (Consider their images under exp restricted to the strip $\frac{\pi}{2} k-\frac{\pi}{2}<y<\frac{\pi}{2}(k+1)+\frac{\pi}{2}$.) It follows that all of the end rays $\gamma_{c, k}$ with $c \in \mathbb{R}$ and $k \in \mathbb{Z}$ are among the representatives of a single (infinite rank) conformal boundary point (which we will call $P_{\infty}$ ).

\section{CyClic ORDER ON $\partial \mathcal{L}$}

Given $P, Q \in \partial \mathcal{L}$, a trail from $P$ to $Q$ is a simple curve formed by concatenating an end ray representing $P$ with a piecewise null arc followed by an end ray representing $Q$. If $\Gamma$ is a trail from $P$ to $Q$ (with $P \neq Q$ ), $S \backslash \operatorname{tr}(\Gamma)$ has two components, $R_{\Gamma}$ (the one to the right of $\Gamma$ ) and $L_{\Gamma}$. Define the $\partial \mathcal{L}$-interval $(P, Q)$ as the set of all conformal boundary points other than $P$ and $Q$ which have end ray representatives contained entirely in $R_{\Gamma}$. It is intuitively clear that the set so obtained does not depend on the choice of trail $\Gamma$, but only on the initial and final points $P$ and $Q$. A proof appears in [11. That there exists a trail connecting any given pair of distinct points of $\partial \mathcal{L}$ follows from Lemma 15 in [12] and the fact that any two points

of $\mathcal{L}$ may be joined by a piecewise-null arc. Lemma 1 lists the most fundamental 
properties of $\partial \mathcal{L}$-intervals. The reader is encouraged to make a comparison with the H-intervals of the definite metric case as defined on pp. 58-60 in [7].

Lemma 1. Given $P, Q, R, S \in \partial \mathcal{L}$ with $P \neq Q$,

(a) $\quad(P, Q) \neq \emptyset$,

(b) $\quad(P, Q) \cap(Q, P)=\emptyset$,

(c) $(P, Q) \cup(Q, P)=\partial \mathcal{L} \backslash\{P, Q\}$,

(d) if $R \in(P, Q)$, then $Q \in(R, P)$, and

(e) if $R \in(P, Q)$ and $Q \in(P, S)$, then $R \in(P, S)$.

To see that $(P, Q)$ is not empty, choose a trail $\Gamma=\gamma_{1}+\cdots+\gamma_{n}$ from $P$ to $Q$, where $\gamma_{1}, \gamma_{n}$ are end rays and $\gamma_{2}, \ldots, \gamma_{n-1}$ are null arcs. Without loss of generality, it may be assumed that $\Gamma$ is minimal in the sense that $n$ is as small as possible. If $\Gamma$ makes a turn to the left at some vertex $V$, then the end rays emanating from $V$ which immediately enter $R_{\Gamma}$ are forced, by the minimality of $\Gamma$, not only to remain in $R_{\Gamma}$ but also to represent conformal boundary points distinct from $P$ and $Q$. Suppose $\Gamma$ does not make any turns to the left. For each point $S$ on $\gamma_{i}$ which is not a vertex of $\Gamma$, consider the end ray $\alpha$ emanating from $S$ which immediately enters $R_{\Gamma}$. Since $\Gamma$ is minimal, either $\alpha$ represents a point of $(P, Q)$, or $i \geq 3$ and $\alpha$ meets $\gamma_{i-2}$, or $i \leq n-2$ and $\alpha$ meets $\gamma_{i+2}$. The supposition that the first case never holds leads to a contradiction. The contradiction is relatively easy to obtain after observing that $n$ must (under the supposition) be at least 4 , and the joint span of the null lines on which $\gamma_{2}$ and $\gamma_{3}$ sit may be conformally embedded in a bounded subspace of $E^{2,1}$. (See Lemma 23 in [12].) Part (c) follows from Lemma 15 in [12]. Parts (b), (d) and (e) are intuitively compelling. The proofs are repetitive and are therefore omitted.

Let $[P, Q]=(P, Q) \cup\{P, Q\}$. The results in Lemma 1 imply

Lemma 2. Given $P, Q, R, S \in \partial \mathcal{L}$, with $P \neq Q$ and $R \neq S$,

(a) if $R \in(P, Q)$ and $S \notin[P, Q]$, then $Q \in(R, S)$,

(b) if $R, S \in[P, Q]$, then $[R, S] \subset[P, Q]$ or $[S, R] \subset[P, Q]$,

(c) if $R \in[P, Q]$ and $S \notin[P, Q]$, then $Q \in[R, S]$ and $P \in[S, R]$,

(d) if $[R, S] \not \subset[P, Q]$ and $P, Q \notin[R, S]$, then $[P, Q] \cap[R, S]=\emptyset$, and

(e) if $[R, S] \subset[P, Q]$, then $R \in[P, S]$ and $S \in[R, Q]$.

The key behind teasing Lemma 2 out of Lemma 1 is the following perspective. For each conformal boundary point $P$ define the relation $<_{P}$ on $\partial \mathcal{L} \backslash\{P\}$ by $R<_{P} Q$ if $R \in(P, Q)$. For each $P \in \partial \mathcal{L}$ the relation $<_{P}$ is a linear order on $\partial \mathcal{L} \backslash\{P\}$. Furthermore if $P, Q, R$ and $S$ are distinct elements of $\partial \mathcal{L}$, then $R<_{Q} S$ if and only if $Q<_{P} R<_{P} S, R<_{P} S<_{P} Q$ or $S<_{P} Q<_{P} R$.

The topology on $\overline{\mathcal{L}}=\mathcal{L} \cup \partial \mathcal{L}$ in 12 is equivalent to the one obtained by taking $\mathcal{B}=\left\{R_{\Gamma} \cup(P, Q): \Gamma\right.$ is a trail from $P$ to $\left.Q\right\}$ as a subbase. Let $C$ be a countable dense subset of the surface $S$. The set $D$ of conformal boundary points represented by end rays that lie along null lines which intersect $C$ is also countable. The proof above which shows that $(P, Q)$ is nonempty for distinct $P, Q \in \partial \mathcal{L}$ may be readily adapted to show that $(P, Q)$ contains some element of $D$. It follows that $\partial \mathcal{L}$ (with the subspace topology inherited from $\overline{\mathcal{L}}$ ) is separable. Henceforth $\partial \mathcal{L}$ is presumed to be endowed with this subspace topology. 


\section{Completing the boundary}

For many simply connected Lorentz surfaces the conformal boundary seems to be topologically pathological. In [5], Kulkarni introduces the phrase wild at infinity to describe any Lorentz surface whose conformal boundary is not a topological 1manifold. For example $\mathcal{L}_{\infty}$ is wild at infinity since $\partial \mathcal{L}_{\infty}$ is not locally connected at $P_{\infty}$. Even the Minkowski plane itself has a noncompact conformal boundary with four connected components. This paper shows that the conformal boundary of a simply connected Lorentz surface is always homeomorphic to a dense subspace of the circle.

The sequence of intervals $\left\{\left[P_{n}, Q_{n}\right]\right\}_{n=1}^{\infty}$ is called nested and shrinking if $\left[P_{n}, Q_{n}\right]$ $\supset\left[P_{n+1}, Q_{n+1}\right], P_{n} \neq P_{n+1}, Q_{n} \neq Q_{n+1}$ for $n=1,2, \ldots$ and $\bigcap_{n=1}^{\infty}\left[P_{n}, Q_{n}\right]$ contains at most one point. Define the relation $\simeq$ on the set of all nested, shrinking sequences of $\partial \mathcal{L}$-intervals as follows. Write $\left\{\left[P_{n}, Q_{n}\right]\right\} \simeq\left\{\left[R_{n}, S_{n}\right]\right\}$ if, for any $m \in \mathbb{Z}^{+}$there exists $l \in \mathbb{Z}^{+}$such that $\left[R_{l}, S_{l}\right] \subset\left[P_{m}, Q_{m}\right]$. Reflexitivity and transitivity of $\simeq$ are trivial. It is also not very hard to show (using Lemmas 1 and 2) that $\simeq$ is symmetric. Define the space of conformal boundary elements $\partial^{c} \mathcal{L}$ as the set of equivalence classes of $\simeq$. (See the analogous developments involving H-intervals on pp. 60-62 of [7]. In particular, compare the definition of $\simeq$ above with Theorem 2.14, and Corollary 1 Lemma 4 and Proposition 1 below with Theorem 2.15.)

Lemma 3. If $\left\{\left[P_{n}, Q_{n}\right]\right\}$ and $\left\{\left[R_{n}, S_{n}\right]\right\}$ represent distinct points of $\partial^{c} \mathcal{L}$, then there exists $N$ such that $\left[P_{N}, Q_{N}\right]$ and $\left[R_{N}, S_{N}\right]$ are disjoint.

Proof. Since $\left\{\left[P_{n}, Q_{n}\right]\right\} \neq\left\{\left\{\left[R_{n}, S_{n}\right]\right\}\right.$ there exists $m \in \mathbb{Z}^{+}$such that $\left[R_{l}, S_{l}\right] \not \subset$ $\left[P_{m}, Q_{m}\right]$ for all $l \in \mathbb{Z}^{+}$. We can assume that $\bigcap\left[R_{l}, S_{l}\right]$ does not equal $\left\{P_{m}\right\}$ or $\left\{Q_{m}\right\}$ (by replacing $m$ with $m+1$ if necessary). Thus there exists $k \in \mathbb{Z}^{+}$such that $P_{m}, Q_{m} \notin\left[R_{k}, S_{k}\right]$. By Lemma $2[\mathrm{~d}),\left[P_{m}, Q_{m}\right] \cap\left[R_{k}, S_{k}\right]=\emptyset$. The result follows with $N=\max \{m, k\}$.

Corollary 1. If $\bigcap_{n=1}^{\infty}\left[P_{n}, Q_{n}\right]=\{P\}=\bigcap_{n=1}^{\infty}\left[R_{n}, S_{n}\right]$ for nested shrinking sequences $\left\{\left[P_{n}, Q_{n}\right]\right\}$ and $\left\{\left[R_{n}, S_{n}\right]\right\}$, then $\left\{\left[P_{n}, Q_{n}\right]\right\} \simeq\left\{\left[R_{n}, S_{n}\right]\right\}$.

The separability of $\partial \mathcal{L}$ (noted above) yields the following lemma.

Lemma 4. Given $P \in \partial \mathcal{L}$ there exists a nested shrinking sequence of intervals $\left\{\left[P_{n}, Q_{n}\right]\right\}$ with $\bigcap_{n=1}^{\infty}\left[P_{n}, Q_{n}\right]=\{P\}$.

Proof. Choose points $R, S \in \partial \mathcal{L}$ such that $R, S$ and $P$ are distinct. By Lemma I(c), $P \in(R, S)$ or $P \in(S, R)$. In the former case, let $P_{1}=R$ and $Q_{1}=S$. Otherwise let $P_{1}=S$ and $Q_{1}=R$. Since $\partial \mathcal{L}$ is separable, there exist sequences of points $\left\{R_{i}\right\}_{i=1}^{\infty}$ and $\left\{S_{i}\right\}_{i=1}^{\infty}$ which have dense images in $\left(P_{1}, P\right)$ and $\left(P, Q_{1}\right)$, respectively. For $n>1$, choose $P_{n}$ from $\left(P_{n-1}, P\right) \cap \bigcap_{i=1}^{n}\left(R_{i}, P\right)$ and $Q_{n}$ from $\left(P, Q_{n-1}\right) \cap \bigcap_{i=1}^{n}\left(P, S_{i}\right)$. The resulting sequence $\left\{\left[P_{n}, Q_{n}\right]\right\}_{n=1}^{\infty}$ is easily seen to have the required properties.

The preceding corollary and lemma yield a natural injection $\partial \mathcal{L} \stackrel{i}{\hookrightarrow} \partial^{c} \mathcal{L}$, so that $\partial \mathcal{L}$ may be identified as a subspace of $\partial^{c} \mathcal{L}$. Points in $\partial^{c} \mathcal{L} \backslash \partial \mathcal{L}$ may be assigned rank 0 . Intervals of boundary elements may be defined as follows. Suppose $P, R$ and $T$ are distinct points of $\partial^{c} \mathcal{L}$ represented by $\left\{\left[P_{n}, Q_{n}\right]\right\},\left\{\left[R_{n}, S_{n}\right]\right\}$ and $\left\{\left[T_{n}, U_{n}\right]\right\}$, respectively. By Lemma 3 we may choose $N$ such that $\left[P_{N}, Q_{N}\right],\left[R_{N}, S_{N}\right]$ and $\left[T_{N}, U_{N}\right]$ are pairwise disjoint. The interval $(P, T)^{c}$ contains the point $R$ if $R_{N} \in$ $\left(P_{N}, T_{N}\right)$. This definition is independent of the choice of representatives of $P, R$ and 
$T$ as well as of the choice of $N$. For $P, Q \in \partial \mathcal{L},(P, Q)=(P, Q)^{c} \cap \partial \mathcal{L}$. Notice that if linear orders are introduced on $\partial^{c} \mathcal{L}$ by analogy with the linear orders on $\partial \mathcal{L}$ defined above, $<_{P}$ on $\partial^{c} \mathcal{L}$ extends $<_{P}$ on $\partial \mathcal{L}$ for each $P \in \partial \mathcal{L}$. The set $\mathcal{L}^{c}=\mathcal{L} \cup \partial^{c} \mathcal{L}$ may be topologized so that $\overline{\mathcal{L}}$ is a subspace. Simply take $\mathcal{B}^{c}=\left\{R_{\Gamma} \cup(P, Q)^{c}: P, Q \in \partial \mathcal{L}, \Gamma\right.$ is a trail from $P$ to $Q\}$ as a subbase. It is readily seen that $\partial^{c} \mathcal{L}$ (with the subspace topology inherited from $\mathcal{L}^{c}$ ) contains $\partial \mathcal{L}$ as a dense subset. It should also be noted that the map $\partial f: \partial \mathcal{L} \rightarrow \partial \hat{\mathcal{L}}$ induced by a conformal equivalence $f: \mathcal{L} \rightarrow \hat{\mathcal{L}}$ between the simply connected Lorentz surfaces $\mathcal{L}$ and $\hat{\mathcal{L}}$ takes nested, shrinking sequences of $\partial \mathcal{L}$-intervals to nested, shrinking sequences of $\partial \hat{\mathcal{L}}$-intervals in a way that respects the relation $\simeq$. Thus $f$ induces a map on the conformal boundary elements $\partial^{c} f: \partial^{c} \mathcal{L} \rightarrow \partial^{c} \hat{\mathcal{L}}$.

Proposition 1. The extended boundary $\partial^{c} \mathcal{L}$ is homeomorphic to the circle.

Proof. Begin by defining in stages a map $f: \partial^{c} \mathcal{L} \rightarrow[0,2 \pi)$. Let $\left\{P_{n}\right\}_{n=1}^{\infty}$ be a sequence of distinct points of $\partial \mathcal{L}$ whose image is dense in $\partial \mathcal{L}$. Define $f\left(P_{1}\right)=0$ and $f\left(P_{2}\right)=\pi$. Extend $f$ inductively as follows. Suppose $f$ has been defined on $\left\{P_{1}, \ldots, P_{n}\right\}$ with $n \geq 2$ so that $f:\left(\left\{P_{2}, \ldots, P_{n}\right\},<_{P_{1}}\right) \rightarrow((0,2 \pi),<)$ is order preserving. If we wish to define $f$ at $P_{n+1}$ so that $f:\left(\left\{P_{2}, \ldots, P_{n+1}\right\},<_{P_{1}}\right) \rightarrow((0,2 \pi),<)$ is order preserving, the possible images of $P_{n+1}$ constitute one of the connected components of $(0,2 \pi) \backslash\left\{f\left(P_{2}\right), \ldots, f\left(P_{n}\right)\right\}$. Define $f\left(P_{n+1}\right)$ to be the midpoint of that distinguished component. Since $f:\left(\left\{P_{n}: n=2,3, \ldots\right\},<_{P_{1}}\right) \rightarrow((0,2 \pi),<)$ is order preserving it is one-to-one. The image $f\left(\left\{P_{n}: n=2,3, \ldots\right\}\right)$ is dense in $(0,2 \pi)$.

Suppose $P^{*} \in \partial^{c} \mathcal{L} \backslash\left\{P_{n}: n=1,2, \ldots\right\}$. Define $f\left(P^{*}\right)=\sup \left\{f\left(P_{n}\right): P_{n}<_{P_{1}} P^{*}\right\}$. The map $f:\left(\partial^{c} \mathcal{L} \backslash\left\{P_{1}\right\},<_{P_{1}}\right) \rightarrow((0,2 \pi),<)$ is order preserving and onto.

The composition $e^{i f}: \partial^{c} \mathcal{L} \rightarrow S^{1}$ is a homeomorphism.

In 4, Klarreich uses the map $f$ of Proposition 1 to characterize the class of simply connected Lorentz surfaces whose conformal boundaries are $C^{0}$ smoothable.

The analogy between the procedure used above to pass from Kulkarni's conformal boundary to the space of conformal boundary elements and Carathéodory's construction of prime ends may be summarized as follows. End rays here take the place of Jordan half-intervals, while Kulkarni's boundary points are the analog of accessible points and conformal boundary elements are the analog of prime ends. The classification of prime ends into 4 kinds is well known. We suggest the following classification of conformal boundary elements in the indefinite metric case. A boundary element in $\partial^{c} \mathcal{L}$ is of the first kind if it corresponds to a point of $\partial \mathcal{L}$. If a rank 0 boundary element is isolated in $\partial^{c} \mathcal{L} \backslash \partial \mathcal{L}$, then it is of the second kind. Otherwise it is of the third kind. For example, the subspace of $E^{2,1}$ above the graph of the greatest integer function has two boundary elements of the third kind, countably many of the second kind, and uncountably many of the first kind.

\section{REFERENCES}

1. C. Carathéodory, Über die Begrenzung einfach zusammenhängender Gebiete, Math. Ann. 73 (1913), 323-370.

2. G. M. Goluzin, Geometric Theory of Functions of a Complex Variable, Vol. 26: Translations of Mathematical Monographs, AMS, 1969. MR 40:308

3. H. Hamburger, Über Kurvennetze mit isolierten Singularitäten auf geschlossen Flächen, Math. Z. 19 (1924), 50-66.

4. N. Klarreich, Smoothability of the conformal boundary of a Lorentz surface implies 'global smoothability', to appear in Geometriae Dedicata. 
5. R. Kulkarni, An analogue of the Riemann mapping theorem for Lorentz metrics, Proc. R. Soc. Lond. A 401 (1985), 117-130. MR 87e:53108

6. F. Luo and R. Stong, An analogue of the Riemann mapping theorem for Lorentz metrics: Topological embedding of a twice foliated disc into the plane, Math Ann 309 (1997), 359-373.

7. A. Markushevich, Theory of Functions of a Complex Variable, Chelsea, 1985. MR 56:3258

8. R. Smyth and T. Weinstein, Conformally homeomorphic Lorentz surfaces need not be conformally diffeomorphic, Proc. Amer. Math. Soc. 123 (1995), 3499-3506. MR 96a:53083

9. R. Smyth and T. Weinstein, How many Lorentz surfaces are there?, Topics in Geometry: In memory of Joseph D'Atri, S. Gindikin, ed., Birkhauser Verlag, 1996. MR 97c:53107]

10. R. Smyth, Uncountably many $C^{0}$ conformally distinct Lorentz surfaces and a finiteness theorem, Proc. Amer. Math. Soc. 124 (1996), 1559-1566. MR 96g:53098

11. R. Smyth, Characterization of Lorentz surfaces via the conformal boundary, Ph.D. Thesis, Rutgers University, 1995.

12. T. Weinstein, An Introduction to Lorentz Surfaces, de Gruyter, 1996. MR 98a:53104

Department of Natural Science, Mathematics and Computer Science, Georgian Court College, Lakewood, New Jersey 08701

E-mail address: smythr@georgian.edu 